\title{
Relationship between morphology, rheology and polygalacturonase production by Aspergillus sojae ATCC 20235 in submerged cultures
}

\author{
Nihan Gögus, Canan Tari*, Selale Oncü, Sevcan Unluturk, Figen Tokatli \\ Department of Food Engineering, Izmir Institute of Technology, Urla, Izmir 35430, Turkey
}

Received 5 January 2006; received in revised form 14 August 2006; accepted 25 September 2006

\begin{abstract}
A full factorial statistical design, with the factors of, two taxonomically different strains, seven types of seed culture formulations (slants) and two types of fermentation media were used to investigate the effect of these parameters on the morphology and polygalacturonase production. The rheology of the final fermentation medium was analyzed and appropriate mathematical model was applied to calculate suspension viscosity. It was found that most fermentation broths showed non-Newtonian flow behavior. According to statistical analysis, factors of strain types and fermentation media and the interaction between them were found significant on the enzyme activity. The effect of seed culture formulations (slants) were found insignificant at the significance level of $1 \%$. Interaction of slants with strain types and fermentation media were also found insignificant. Considering the morphology of the final culture, Aspergillus sojae with the desired pellet morphology in a complex media, inoculated with a seed culture prepared from molasses resulted in maximum polygalacturonase enzyme activity $(0.2 \mathrm{U} / \mathrm{ml})$ and lowest suspension viscosity with a broth rheology close to Newtonian flow behavior.
\end{abstract}

(C) 2006 Elsevier B.V. All rights reserved.

Keywords: Aspergillus sojae; Rhizopus oryzae; Polygalacturonase; Submerged fermentation; Fungal morphology; Rheology

\section{Introduction}

Submerged fungal fermentations are widely used in the production of enzymes, antibiotics and organic acids, which have lots of applications in the food, medicine, pharmaceutical, chemical and textile industry [1]. However, their filamentous growth characteristic creates a number of process engineering problems attributed to the morphological change accounted during the fermentation process in large scales. It is well documented that the fungal culture exhibits two major morphologies observed as pellet or mycelia, which are very much determined by several environmental and genetic factors [2,3]. These are; type of the strain, $\mathrm{pH}$ and composition of the media, inoculation ratio, type of the inoculum, agitation speed, aeration rate, feeding rate and genetic factors of the culture [4]. In industrial applications pellet morphology is usually preferred in fermentations and in downstream processing due to the non-viscous (Newtonian) rheology of the broth $[5,6]$. In such fermentations, the mass transfer of oxygen and nutrients is considerably better and the

\footnotetext{
* Corresponding author. Tel.: +90 232 7506316; fax: +90 2327506196.

E-mail addresses: canantari@iyte.edu.tr, ctari7@yahoo.com (C. Tari).
}

subsequent separation of the pellets from the medium is simpler [4]. Since agitation and aeration is also much easier in such a system, the power input therefore the operating cost is lower. However, in fermentations where the mycelia form is dominant and the cell growth and productivity might be higher, the broth is much more viscous (non-Newtonian), resulting in heterogeneous and stagnant non-mixed zone formations, that are harder and more expensive to operate [7]. Hence, the balance between these parameters has to be established in an effective manner. This is only possible by controlling the morphology of the culture, which effects productivity and results into rheological changes of the broth. Therefore, the relationship between morphology, rheology and product formation and the factors influencing these needs to be fully investigated.

Although several studies have been conducted on the rheology of fermentation broths carried out in fermentors, there is lack of research on the flow behavior of fermentation broth conducted in shake flasks, which is mostly used in initial optimization studies of important parameters that play significant role in scale-up processes [8]. With this perspective, the scope of this paper was to take polygalacturonase enzyme production from Aspergillus sojae ATCC 20235 (from here on A. sojae) and Rhizopus oryzae ATCC 4858 (from here on $R$. oryzae) known 
with their different taxonomies as a model to unveil the problems associated with the morphology and broth rheology, and at the same time initiate a study for the optimization of the production of an industrially important enzyme (polygalacturonase) which has many applications in the food, textile, paper industries and waste water treatment $[9,10]$.

Initial sub-culturing step (preparation of seed culture) with respect to its formulation and cultivation conditions, which has been mostly ignored in the industrial applications, is believed to have major effect on spore production and on the final morphology, rheology and product yield of the fermentation process. However, there is no literature to best of our knowledge on the media compositions used for the preparations of the seed cultures and their effects. Although, the actual fermentation media composition, has been always an important parameter worked on by many scientist and there is an enormous literature on the optimization of the formulations for different strains and products, there is unfortunately no available literature on its interaction with seed media compositions [11-13]. Therefore, in this study the aim was also to investigate the effect and the interaction of fungal strain, seed and fermentation media formulations on the mentioned parameters using full factorial experimental design. As it is known full factorial experimental design is the best alternative to the conventional one at a time experimental strategy, since in a well-designed experimental environment, all factors effects and most importantly their interaction effects can be identified.

Although there are several studies on $R$. oryzae in the production of polygalacturonase, which is mostly taken as a reference for comparison in this study, there is no available literature report on the polygalacturonase production by $A$. sojae in deep tank and solid-state fermentation $[14,15]$. So far $A$. sojae is known to be used in the production of a well-known Japanese food koji, by means of solid-state fermentation [16]. Therefore, this paper will be one of initial studies working in this field and be a new reference point for the microbiology area and the enzyme industry with respect to the new application of this organism (A. sojae) in submerged fermentation.

\section{Materials and methods}

\subsection{Microorganisms}

A. sojae ATCC 20235 and $R$. oryzae ATCC 4858 were purchased from Procochem Inc., an international distributor of ATCC (American Type of Culture Collection) in Europe. These were obtained in lyophilized form and were propagated on YME agar slant medium containing, malt extract at $10 \mathrm{~g} / \mathrm{l}$, yeast extract at $4 \mathrm{~g} / \mathrm{l}$, glucose at $4 \mathrm{~g} / \mathrm{l}$ and agar at $20 \mathrm{~g} / \mathrm{l}$ concentrations incubated at $30{ }^{\circ} \mathrm{C}$ until well sporulated (1 week). Stock cultures of these strains were prepared with $20 \%$ glycerol water and stored at $-80{ }^{\circ} \mathrm{C}$.

\subsection{Growth medium}

For the seed medium, seven different media formulations as described in Table 1 were used. Initially, the frozen stock
Table 1

Seed media formulations used in the preparation of spore suspensions

\begin{tabular}{|c|c|}
\hline Media code & Composition \\
\hline Yeast malt extract agar (YME) & $\begin{array}{l}\text { Malt extract }(10 \mathrm{~g} / \mathrm{l}) \text {; yeast extract }(4 \mathrm{~g} / \mathrm{l}) \\
\text { glucose }(4 \mathrm{~g} / \mathrm{l}) ; \text { agar }(20 \mathrm{~g} / \mathrm{l})\end{array}$ \\
\hline Yeast malt pepton agar (YMP) & $\begin{array}{l}\text { Malt extract }(3 \mathrm{~g} / \mathrm{l}) \text {; yeast extract }(3 \mathrm{~g} / \mathrm{l}) \text {; } \\
\text { pepton }(3 \mathrm{~g} / \mathrm{l}) \text {; glycerol }(20 \mathrm{~g} / \mathrm{l}) \text {; agar } \\
(20 \mathrm{~g} / \mathrm{l})\end{array}$ \\
\hline Molasses medium (M) & $\begin{array}{l}\text { Glycerol }(45 \mathrm{~g} / \mathrm{l}) ; \text { pepton }(18 \mathrm{~g} / \mathrm{l}) ; \\
\text { molasses }(45 \mathrm{~g} / \mathrm{l}) ; \mathrm{NaCl}(5 \mathrm{~g} / \mathrm{l}) ; \\
\mathrm{FeSO}_{4} \cdot 7 \mathrm{H}_{2} \mathrm{O}(15 \mathrm{mg} / \mathrm{l}) ; \mathrm{KH}_{2} \mathrm{PO}_{4} \\
(60 \mathrm{mg} / \mathrm{l}) ; \mathrm{MgSO}_{4}(50 \mathrm{mg} / \mathrm{l}) ; \\
\mathrm{CuSO}_{4} \cdot 5 \mathrm{H}_{2} \mathrm{O}(12 \mathrm{mg} / \mathrm{l}) ; \mathrm{MnSO}_{4} \cdot \mathrm{H}_{2} \mathrm{O} \\
(15 \mathrm{mg} / \mathrm{l}) ; \operatorname{agar}(20 \mathrm{~g} / \mathrm{l})\end{array}$ \\
\hline ATCC media $^{\mathrm{a}}$ & $\begin{array}{l}\text { Corn meal }(5 \mathrm{~g} / \mathrm{l}) \text {; yeast extract }(2 \mathrm{~g} / \mathrm{l}) \text {; } \\
\text { casitone }(1 \mathrm{~g} / \mathrm{l}) ; \text { malt extract }(1 \mathrm{~g} / \mathrm{l}) \text {; } \\
\mathrm{MgSO}_{4} \cdot 7 \mathrm{H}_{2} \mathrm{O}(0.5 \mathrm{~g} / \mathrm{l}) ; \mathrm{KH}_{2} \mathrm{PO}_{4} \\
(1.5 \mathrm{~g} / \mathrm{l}) ; \operatorname{agar}(20 \mathrm{~g} / \mathrm{l})\end{array}$ \\
\hline Defatted soy bean (DSM) & $\begin{array}{l}\text { Defatted soybean }(5 \mathrm{~g} / \mathrm{l}) ; \text { malt extract } \\
(20 \mathrm{~g} / \mathrm{l}) ; \mathrm{KH}_{2} \mathrm{PO}_{4}(10 \mathrm{~g} / \mathrm{l}) ; \text { agar }(20 \mathrm{~g} / \mathrm{l})\end{array}$ \\
\hline Complex medium (CM) & $\begin{array}{l}\text { Glucose }(25 \mathrm{~g} / \mathrm{l}) \text {; matrin } 50(25 \mathrm{~g} / \mathrm{l}) \text {; corn } \\
\text { steep liquor }(2.5 \mathrm{~g} / \mathrm{l}) \text {; pepton }(2.5 \mathrm{~g} / \mathrm{l}) \text {; } \\
\text { disodium phosphate }(3.2 \mathrm{~g} / \mathrm{l}) \text {; } \\
\text { monosodium phosphate }(3.3 \mathrm{~g} / \mathrm{l}) \text {; agar } \\
(20 \mathrm{~g} / \mathrm{l})\end{array}$ \\
\hline Defined medium (DM) & $\begin{array}{l}\text { Glucose }(50 \mathrm{~g} / \mathrm{l}) ; \text { yeast extract }(2 \mathrm{~g} / \mathrm{l}) ; \\
\mathrm{KH}_{2} \mathrm{PO}_{4}(0.6 \mathrm{~g} / \mathrm{l}) ; \mathrm{MgSO}_{4} \cdot 7 \mathrm{H}_{2} \mathrm{O} \\
(0.5 \mathrm{~g} / \mathrm{l}) ; \mathrm{ZnSO}_{4} \cdot 7 \mathrm{H}_{2} \mathrm{O}(0.0176 \mathrm{~g} / \mathrm{l}) ; \\
\mathrm{FeSO}_{4} \cdot 7 \mathrm{H}_{2} \mathrm{O}(0.498 \mathrm{mg} / \mathrm{l}) ; \text { bacto agar } \\
(20 \mathrm{~g} / \mathrm{l})\end{array}$ \\
\hline
\end{tabular}

a ATCC media: for $R$. oryzae this is corn meal agar (purchased by Bacto) and for A. sojae it is the one stated in the column.

cultures were inoculated on YME agar and incubated for 1 week at $30^{\circ} \mathrm{C}$ for activation. After this period a single isolate of each strain was inoculated on five slants for each media composition as indicated in Table 1 and incubated for another week at the same temperature. Following the incubation period, $5 \mathrm{ml}$ of $0.01 \%(\mathrm{v} / \mathrm{v})$ tween 80 solution was added into each slant and harvested into empty sterile falcon tube. The content of five slants from each group was gathered into one tube and tested for sterility, viability and spore count. The spore counts were performed using Thoma bright line hemacytometer (Marienfield, Germany). The suspensions were stored at $4{ }^{\circ} \mathrm{C}$ and used as inoculum for the fermentation process, which was performed according to the full factorial experimental design.

\subsection{Production medium and experimental design}

Full factorial experimental design was used in order to identify important parameters in the screening analysis. The factors were the strain types, production (fermentation) and seed media formulation. As the strain types, A. sojae ATCC 20235 and $R$. oryzae ATCC 4858 were used. Two different fermentation media were used as defined and complex media (see compositions in Table 1 without agar). As seed media formulations, seven different slant media were used; YME, YMP, ATCC, molasses, DSB, CA and DA. Total of 56 shake flasks experiments $(50 \mathrm{ml}$ 
in $250 \mathrm{ml}$ Erlenmeyer) were conducted with two replicates of each factorial combination. These were inoculated with $5 \times 10^{5}$ total spores, coming from the slants prepared in Section 2.2 and incubated for $96 \mathrm{~h}$ at $250 \mathrm{rpm}$ and $30^{\circ} \mathrm{C}$. After this period, each flask was assayed for enzyme activity, fungal morphology and broth rheology. The final $\mathrm{pH}$ of the flasks ranged between 3 and 3.5. Enzyme activity was determined on supernatant obtained after the centrifugation of the broth at $5000 \mathrm{rpm}$ for $15 \mathrm{~min}$. Analysis of data was done by Modde 7 statistical software (Umetrics).

\subsection{Enzyme assay}

Polygalacturunase (PG) activity was assayed according to the procedure given by Panda et al. [17] by using $2.4 \mathrm{~g} / \mathrm{l}$ of polygalacturonic acid as substrate at $\mathrm{pH} 6.6$ and $26^{\circ} \mathrm{C}$. The amount of substrate and enzymes used were 0.4 and $0.086 \mathrm{ml}$, respectively. In this study, one unit of enzyme activity was defined as the amount of enzyme that catalyses the release of $1 \mu \mathrm{mol}$ of galacturonic acid per unit volume of culture filtrate per unit time at standard assay conditions. Galacturonic acid (Sigma, St. Louis, MO) was used as standard for the calibration curve of PGase activity.

activity $(\mathrm{U} / \mathrm{ml})=\frac{\mathrm{mg} \text { of galacturonic acid }}{212.12} \times \frac{1}{20} \times \frac{1}{0.086}$

\subsection{Rheological and morphological measurements}

Rheological properties of fermentation broth were determined by using a programmable rotational viscometer (Brookfield DV II+, Brookfield, Middleboro, MA). Since each of the shake flasks had $50 \mathrm{ml}$ fermentation media, UL adapter and/or a small sample adapter were attached to the viscometer to test small amount of sample. Samples were collected at the beginning and at the end of the incubation period ( 0 and $96 \mathrm{~h})$. But in the samples collected at $96 \mathrm{~h}$, the size of the pellets was generally of the same order of magnitude as the annulus of the adapters. In order to overcome this measurement problem, the measured viscosity of fermentation broth was assumed to be a suspension and corrected by using a mathematical model developed by Metzner [18]. The model for estimating viscosity of dilute suspensions was based on volume fraction of the suspended solids (pellet) $(\phi)$ and the relative viscosity of the suspension, given in Eqs. (2) and (3), where $\eta$ was the viscosity of the suspension and $\eta_{\mathrm{s}}$ was the viscosity of the continuous phase [18]:

$\eta_{\mathrm{r}}=\frac{\eta}{\eta_{\mathrm{s}}}$

$\eta_{\mathrm{r}}=\left[1-\left(\frac{\phi}{A}\right)\right]^{-2}$

In Eq. (3), $A$ was in the range of 0.44 and 0.68 for the crystal and spherical shape particles. For this purpose, the volume and mass of the each sample containing pellet was initially measured and the bulk density of the suspension (fermentation broth) was calculated. Then the sample was filtered and mass and volume measurements were repeated for the filtrate (clear broth). Volume fraction $(\phi)(\mathrm{v} / \mathrm{v})$, pellet percentage $(\%, \mathrm{w} / \mathrm{v})$ and wet pellet density were calculated from these measurements. After, the viscosity of the clear broth, $\eta_{\mathrm{s}}$, was measured by using viscometer.

Pellet morphology was characterized by using image analysis [19]. Pellet particles were analyzed for determination of the number of pellet per given volume and pellet size. The image was captured with a eurocam (Euromax, Holland) mounted on a phase contrast microscope (Novex, Holland). Image analysis was performed with the software package Image-Pro Plus 4.5.1. (Media Cybernetics Inc., Silver Spring, MD, USA). The size of the pellet was quantified using the diameter corresponding to a circular area equivalent to the pellet projected area [20].

\section{Result and discussions}

\subsection{Effect of type of strain and production media on polygalacturonase enzyme activity}

There are number of reports showing that various fungal fermentations require different growth morphologies for optimum product yield [21,19]. For example pelleted growth is preferred for itaconic acid production by Aspergillus terreus, whereas pelleted and filamentous growth has been used for penicillin production industrially. However, only filamentous growth is necessary for maximum pectic enzyme production by Aspergillus niger and for fumaric acid production by Rhizopus arrhizus [7,22,23].

Statistical analysis of full factorial design with the mean values and standard deviations of activity measurements considering the factor variables (type of strain, seed and fermentation media composition) is presented in Tables 2 and 3. Small $p$-values $(p<0.01)$ show that the parameter is significant on the response at the $1 \%$ level of significance. Factors of strain types and fermentation media and the interaction between them were found significant on the enzyme activity, whereas the seed media formulation (slants) was found insignificant. Interaction between strain types and slants, and interaction between fermentation media and slants were also found insignificant $(p$ value $\gg 0.01)$. A. sojae produced almost four-times more polygalacturonase enzyme than $R$. oryzae in defined medium (Fig. 1). However, in the complex medium this difference could not be detected. These results are indicative that, $R$. oryzae has either more nutritional requirements that was not present in defined medium or there were some inhibitor(s), which might have been present initially or formed later.

As it is seen from Figs. 1 and 2, defined medium compared to complex medium was more affected by the slant media composition and was not suitable for the cultivation of $R$. oryzae. On the other hand, complex medium increased the enzyme activity 5 times more for $R$. oryzae, where this increase was only 1.25 times for A. sojae (Fig. 1). Even though the increase was low for $A$. sojae, this was a positive effect. This showed that this organism would perform in spite of limited nutrients, baring the 
Table 2

The mean values and standard deviations of activity measurements in full factorial design

\begin{tabular}{|c|c|c|c|c|c|}
\hline Combination & Strain & Media & Slant & Activity_mean ${ }^{\mathrm{a}}(\mathrm{U} / \mathrm{ml})$ & Activity_stdev ${ }^{\mathrm{a}}(\mathrm{U} / \mathrm{ml})$ \\
\hline 1 & AS & def & YME & 0.237 & 0.023 \\
\hline 2 & RO & def & YME & 0.014 & 0.020 \\
\hline 3 & AS & comp & YME & 0.277 & 0.040 \\
\hline 5 & AS & def & YMP & 0.147 & 0.101 \\
\hline 6 & RO & def & YMP & 0.013 & 0.019 \\
\hline 7 & AS & comp & YMP & 0.246 & 0.070 \\
\hline 10 & RO & def & ATCC & 0.066 & 0.024 \\
\hline 11 & AS & comp & ATCC & 0.280 & 0.054 \\
\hline 12 & RO & comp & ATCC & 0.282 & 0.095 \\
\hline 13 & AS & def & M & 0.244 & 0.033 \\
\hline 14 & RO & def & M & 0.079 & 0.112 \\
\hline 15 & AS & comp & M & 0.258 & 0.042 \\
\hline 20 & RO & comp & DSB & 0.254 & 0.081 \\
\hline 21 & AS & def & $\mathrm{CA}$ & 0.175 & 0.100 \\
\hline 22 & RO & def & $\mathrm{CA}$ & 0.009 & 0.013 \\
\hline 23 & AS & comp & $\mathrm{CA}$ & 0.249 & 0.054 \\
\hline 24 & $\mathrm{RO}$ & comp & $\mathrm{CA}$ & 0.263 & 0.080 \\
\hline 25 & AS & def & DA & 0.216 & 0.069 \\
\hline 26 & $\mathrm{RO}$ & def & DA & 0.000 & 0.000 \\
\hline 27 & AS & comp & DA & 0.281 & 0.083 \\
\hline 28 & RO & comp & $\mathrm{DA}$ & 0.265 & 0.085 \\
\hline
\end{tabular}

${ }^{\text {a }}$ Mean and standard deviations were calculated based on two replications. AS: A. sojae; RO: R. oryzae; def: defined medium; comp: complex medium; explanations for YME, YMP, ATCC, M, DSB, CA and DA given in Table 1.

potential to be a good candidate for industrial use with possibly less expensive nutrient requirements. The enzyme activities obtained in this study were comparable to the literature for various Aspergillus sp and Rhizopus sp. [24,25].

Table 3

Results of full factorial design: effect of factors and interactions on the polygalacturonase enzyme activity

\begin{tabular}{ll}
\hline Response: activity & $p$-Value \\
\hline Factor: strain & \\
$\quad$ AS & $2.06376 \mathrm{e}-006$ \\
RO & $2.06376 \mathrm{e}-006$ \\
Factor: media & \\
$\quad$ def & $8.20811 \mathrm{e}-011$ \\
$\quad$ comp & $8.20811 \mathrm{e}-011$ \\
Factor: seed culture & \\
$\quad$ YME, YMP, ATCC, molasses, DSB, CA, DA & $\gg 0.01$, insignificant \\
Interaction: strain $\times$ media & \\
$\quad$ AS ${ }^{a}$ def & $1.70172 \mathrm{e}-006$ \\
AS ${ }^{\mathrm{a}}$ comp & $1.70172 \mathrm{e}-006$ \\
RO ${ }^{\mathrm{a}}$ def & $1.70172 \mathrm{e}-006$ \\
RO $^{\mathrm{a}}$ comp & $1.70172 \mathrm{e}-006$ \\
Interaction: strain $\times$ seed culture & $\gg 0.01$, insignificant \\
Interaction: media $\times$ seed culture & $\gg 0.01$, insignificant
\end{tabular}

a AS: A. sojae; RO: R. oryzae; def: defined medium; comp: complex medium; explanations for YME, YMP, ATCC, molasses, DSB, CA, DA are given in Table 1.
Considering the morphological parameters, $A$. sojae resulted almost in all runs into pellet morphology with higher enzyme activity, where $R$. oryzae on the contrary exhibited clump morphology only. The enzyme synthesis of $R$. oryzae was not affected by the morphology. However, in the literature there are number of studies [26,27], reporting pellet morphology for Rhizopus spp. Samples exhibiting pellet morphology (in this case only from A. sojae) were analyzed further for morphological and rheological studies, discussed in Section 3.3.

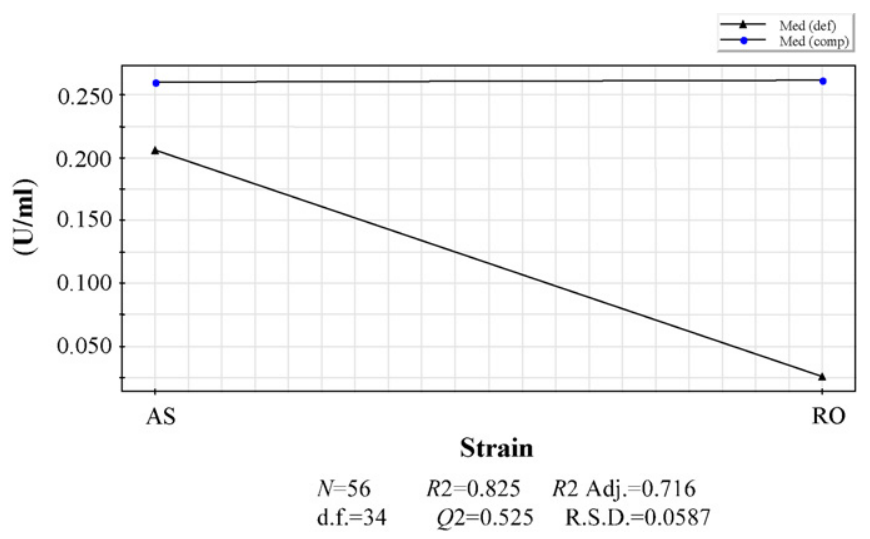

Fig. 1. Result of full factorial design: effect of the interaction of fungal strain and seed culture media on the polygalacturonase enzyme activity (U/ml). AS: Aspergillus sojae; RO: Rhizopus oryzae; def: defined; comp: complex. 


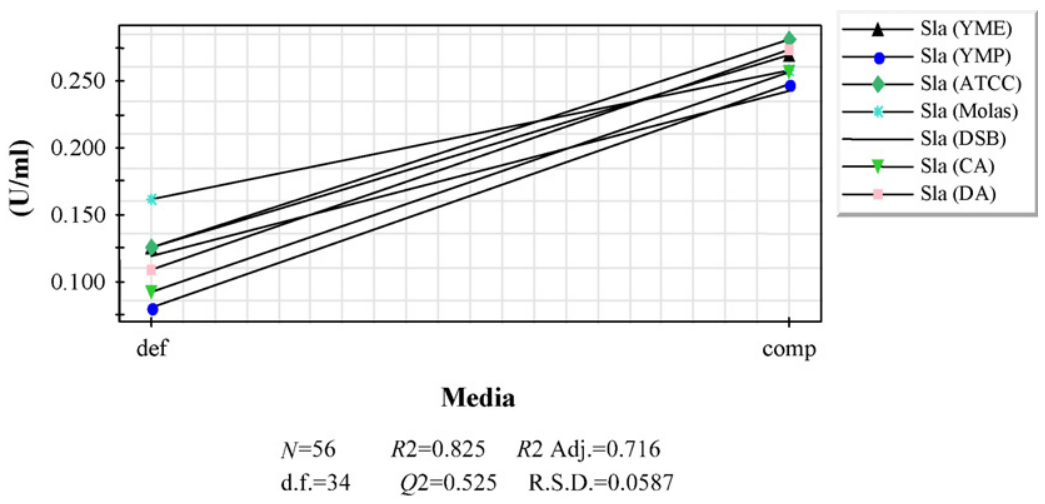

Fig. 2. Result of full factorial design: effect of the interaction of fermentation media and seed culture media (def, defined and comp, complex) on the polygalacturonase enzyme activity $(\mathrm{U} / \mathrm{ml})$. The explanation of the abbreviations are given in Table 1.

\subsection{Effect of seed culture composition on the spore production}

In order to investigate the effect of seed culture composition on spore production, seven different formulations (obtained from literature and based on our experience) as outlined in Table 1 were considered in the preparation of the seed culture media $[5,28]$. The spore counts obtained from these spore suspensions are presented in Fig. 3. As it is observed among all the compositions, the molasses and dry soy bean media promoted more spore growth for A. sojae. However, the least favorable one was with composition suggested by ATCC. The same results were YMP and ATCC media for $R$. oryzae, respectively. It was very interesting to note that for both cultures the media suggested by ATCC was not very favorable. The same problem was also encountered at the initial propagation step when they were obtained in lyophilized forms.

The spore counts of A. sojae, based on the favorable media formulation (molasses for A. sojae and YME for R. oryzae), were almost six times higher than $R$. oryzae under the same incubation conditions. This result indicated that, different strains require different incubation period and conditions, requiring optimization. As a result, considering spore counts and economic feasibility, molasses or defatted soybean media would be the media that could be recommended for activating both cul-

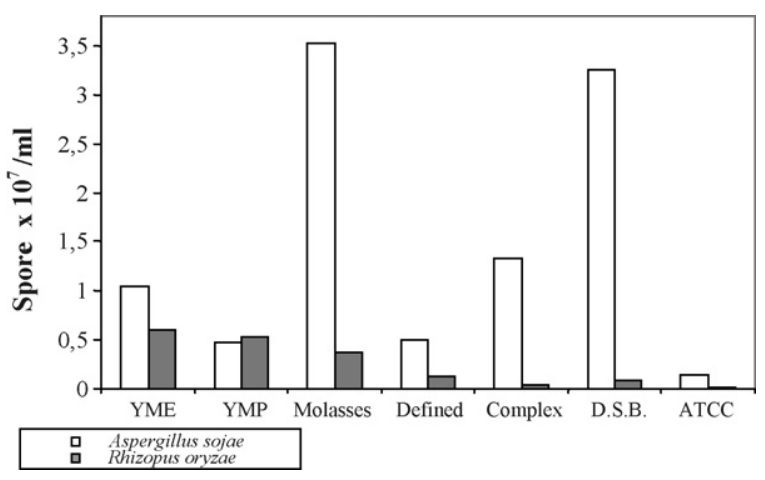

Fig. 3. Spore counts obtained using various seed media formulations by A. sojae and $R$. oryzae. Standard deviation of spore counts of A. sojae varies in a range of $9.62 \mathrm{e}+06$ and $1.98 \mathrm{e}+07$ spore $/ \mathrm{ml}$. Standard deviation of spore counts of $R$. oryzae varies in a range of $9.48 \mathrm{e}+05$ and $2.83 \mathrm{e}+07$ spore $/ \mathrm{ml}$. tures. Furthermore, the effect of the seed media composition was pronounced more in defined media, where molasses seed media resulted in highest enzyme activity also (Fig. 2).

Although, the seed culture composition did not affect the final morphology of $R$. oryzae, it did cause a significant effect on the final morphology of $A$. sojae, where different size and density pellets were observed.

\subsection{Pellet morphology and broth rheology}

In this work, as it is mentioned in previous sections, $R$. oryzae showed a clump type of morphology and $A$. sojae formed pellet type of morphology under the same experimental conditions. As a result, eight fermentation broths (Table 4) which were inoculated with A. sojae and showed pellet morphology were chosen for the comparison of the rheological and morphological properties to decipher the effect of seed culture and the production medium on the enzyme activity.

Rheological behavior of fermentation broths of both filtrated (free of pellet) and suspensions (with pellet) obtained in shake flask experiments are depicted in Fig. 4. There was a tendency towards to non-Newtonian behavior at high shear rates for the filtrated medium. But the recorded values of viscosity were in the range of 1 and $2 \mathrm{cP}$ similar to water, so that this behavior was ignored. Nevertheless, the filtrated broths were Newtonian along the culture. Although the filtrates of different culture media had a lower viscosity, the variation of the filtrated broth viscosity

Table 4

Abbreviations used for the fermentation broth which showed pellet type of morphology

\begin{tabular}{lll}
\hline Seed media & Production media & Abbreviation for fermentation broth \\
\hline YME & Defined & YME (ADYME) \\
YMP & Defined & YMP (ADYMP) \\
ATCC & Defined & ATCC (ADATCC) \\
Molasses & Defined & Molasses (ADM) \\
Molasses & Complex & Molasses (ACM) \\
DSB & Defined & DSB (ADDSB) \\
DSB & Complex & DSB (ACDSB) \\
Complex & Defined & CA (ADCA)
\end{tabular}

a A, D, C refer to A. sojae, defined media, and complex media, respectively. 

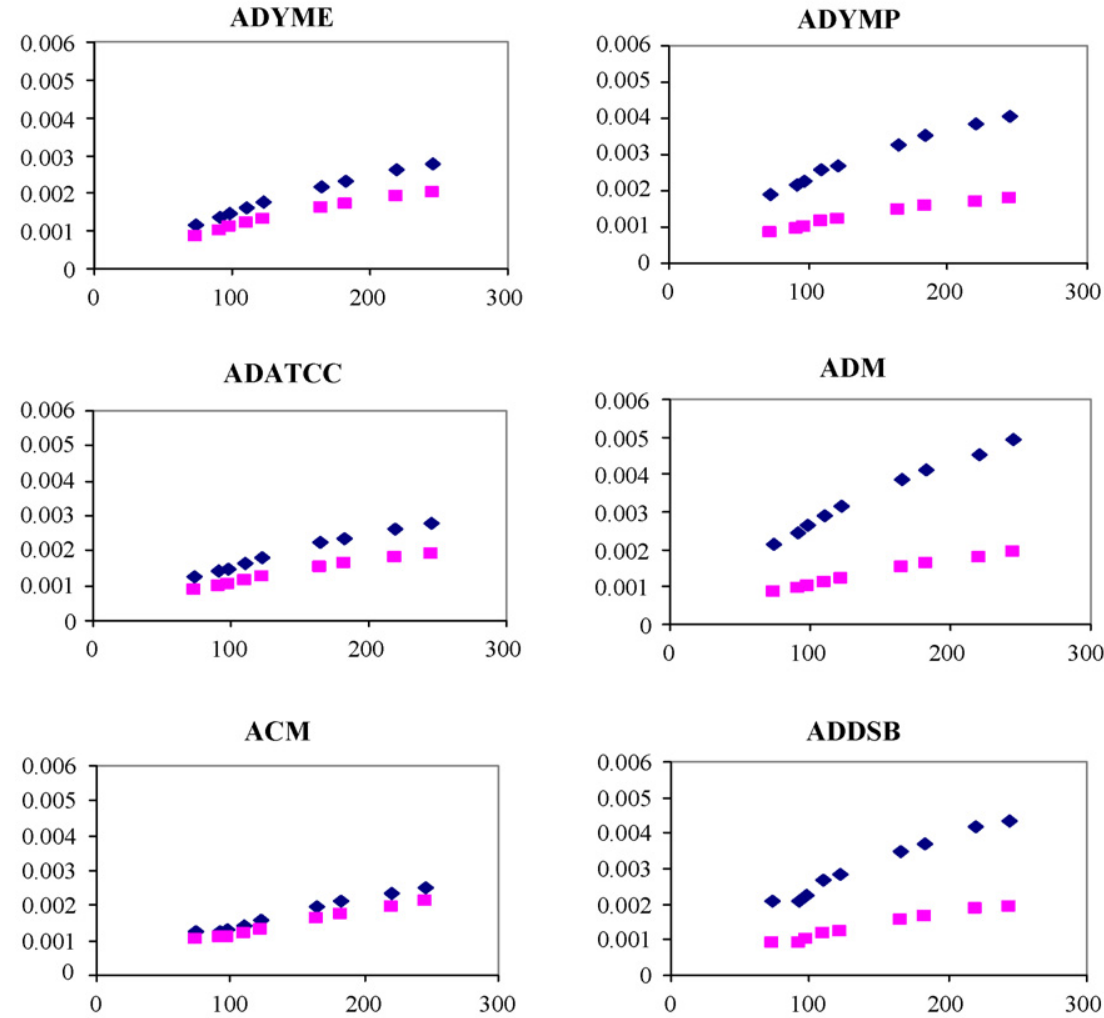

ACDSB
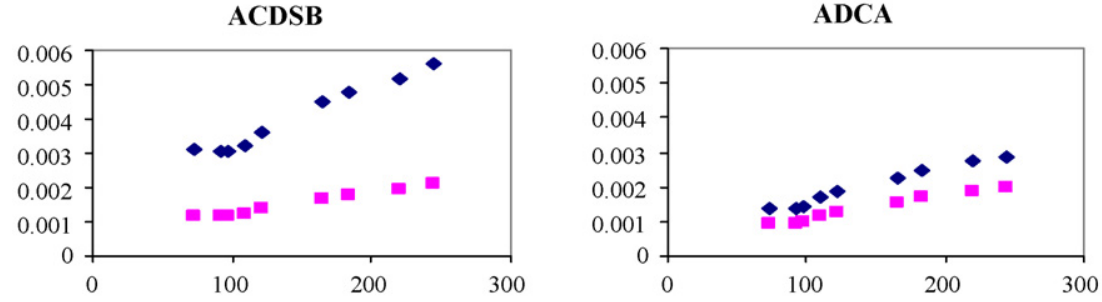

Shear Rate (1/s)

Fig. 4. Rheograms of A. sojae broths obtained from shake flask experiments resulted in pellet type of morphology as a function of shear rate. Rheological behavior of suspensions ( $)$ and pellet-free medium (filtrated broth) (ם). Explanation of the abbreviations are given in Table 4.

among different media formulations was found to be $28 \%$ at low shear rates $\left(73.4 \mathrm{~s}^{-1}\right)$ whereas it was $15 \%$ at high shear rates $\left(245 \mathrm{~s}^{-1}\right)$. The suspension broths from the ADYME, ADATCC, ACM and ADCA cultures carried out in shake flasks were also close to Newtonian with the viscosity values similar to the filtered (pellet-free) medium (Fig. 4). However, non-Newtonian and shear-thickening behavior was more pronounced for the suspensions of ADYMP, ADM, ADDSB, ACDSB [8], indicated that the majority of studies about the fungal fermentation carried out in bioreactors exhibited non-Newtonian and shear-thinning (pseudo-plastic) characteristics. One of the reasons of this difference might be due to the operational conditions (agitation speed, aeration, inoculum size, etc.) carried out in stirred tank reactors and shake flask experiments. The other reason might be the accumulation of extracellular compounds in the medium during fermentation process. Additionally, suspensions of ADYMP, ADM, ADDSB, ACDSB type of seed and production media had higher pellet volume fractions $(\mathrm{v} / \mathrm{v})$, pellet $\%(\mathrm{w} / \mathrm{v})$ and number of pellets per given volume of broths (Table 5). On the other hand, A. sojae formed larger and lesser number of pellets in ADYME, ADATCC, ADCA type of seed and production media. ACM media showed the highest bulk density and lower pellet volume fraction. So, it was shown that all of these morphological parameters might have a significant role on suspension viscosity and broth rheology.

The effect of suspension viscosity on the enzyme activity was also investigated (Fig. 5 and Table 5). A. sojae cultivated from molasses seed media and grown in complex medium (ACM) 
Table 5

Morphological parameters for different fermentation media which showed pellet type of morphology

\begin{tabular}{|c|c|c|c|c|c|}
\hline Media $^{a}$ & $\begin{array}{l}\text { Bulk pellet density } \\
\left(\mathrm{kg} / \mathrm{m}^{3}\right)\end{array}$ & $\begin{array}{l}\text { Number of pellet } \\
\text { (in } 13 \mathrm{ml} \text { sample) }\end{array}$ & $\begin{array}{l}\text { Pellet volume } \\
\text { fraction }(\mathrm{v} / \mathrm{v})\end{array}$ & Pellet \% (w/v) & $\begin{array}{l}\text { Enzyme activity } \\
(\mathrm{U} / \mathrm{ml})\end{array}$ \\
\hline ADYME & 0.9576 & 36 & 0.0854 & 7.7725 & 0.045 \\
\hline ADYMP & 0.9589 & 240 & 0.2000 & 19.0042 & 0.0093 \\
\hline ADM & 0.7568 & 160 & 0.2250 & 16.9570 & 0.052 \\
\hline $\mathrm{ACM}$ & 3.6846 & 103 & 0.0513 & 18.0470 & 0.2073 \\
\hline ADDSB & 0.8599 & 109 & 0.2000 & 14.6803 & 0.0568 \\
\hline
\end{tabular}

${ }^{a}$ Explanations for the abbreviations are given in Table 4.

showed higher enzyme activity and lower suspension viscosity among the eight mediums. Because of the Newtonian behavior and low viscosity properties of ACM, expedited the use of available oxygen and nutrients by the organism, formed pellets with higher bulk density and produced more enzyme in ACM medium.

Morphology of the pellets was also analyzed by applying image processing. Cox and Thomas [19] proposed image analysis methods to characterize pellets based on the presence of central core. They classified the pellets into two groups: smooth and hairy types. Taking this study into account, the morphology of the pellet formed in complex medium was characterized as a spherical shape of particle with a dense core zone surrounded by a fluffy region (similar to hairy type). However, the pellet grown in defined medium had a perfect spherical shape classified as smooth type.

The size of pellets was in the range of 1 and $4 \mathrm{~mm}$ (Fig. 6). Although pellets grown in ADYMP medium had a narrow size distribution, the enzyme activity was the lowest for this medium formulation. This might be due to non-Newtonian rheology,

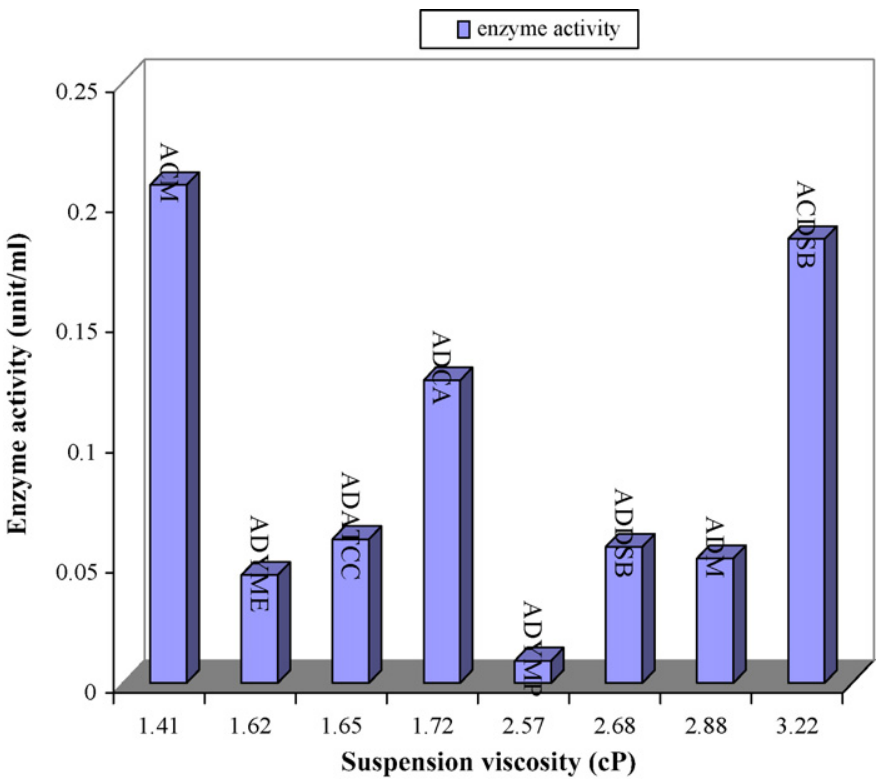

Fig. 5. The effect of suspension viscosity on the polygalacturonase enzyme activity $(\mathrm{U} / \mathrm{ml})$ of $A$. sojae in shake flask experiments resulted in pellet type of morphology. Explanation of the abbreviations are given in Table 4. which complicated the mass transfer characteristics of the suspension broth. On the other hand, the size distribution of pellets formed in ACM medium was close to normal size distribution, indicating the homogeneity of the broth suspension. The high enzyme activity in this medium could be also explained by the Newtonian rheology of the broth.

It has been shown that there is a direct relationship between the rheological and morphological parameters in the case of shake-flask experiments. In stirred tank bioreactors, agitation speed, inoculation ratio etc. are different. Particularly, aeration causes significant changes in broth rheology and morphology. It was observed that there was a tendency to non-Newtonian behavior at high shear rates and ignored in shake flask experiments. But this behavior will become dominant in stirred tank fermentations due to the effect of higher agitation speeds and aeration. It is likely to observe some differences in rheological behavior between bioreactor and the shake flask experiments. But this dissimilarity was already predicted by studying the rheological behavior and morphological parameters in shake flask experiments. This will be taken into account in scaling-up of this process.

As a result, the rheological and morphological measurements proved that, $A$. sojae as a strain, cultivated using molasses media as seed media and carrying out the main fermentation in complex

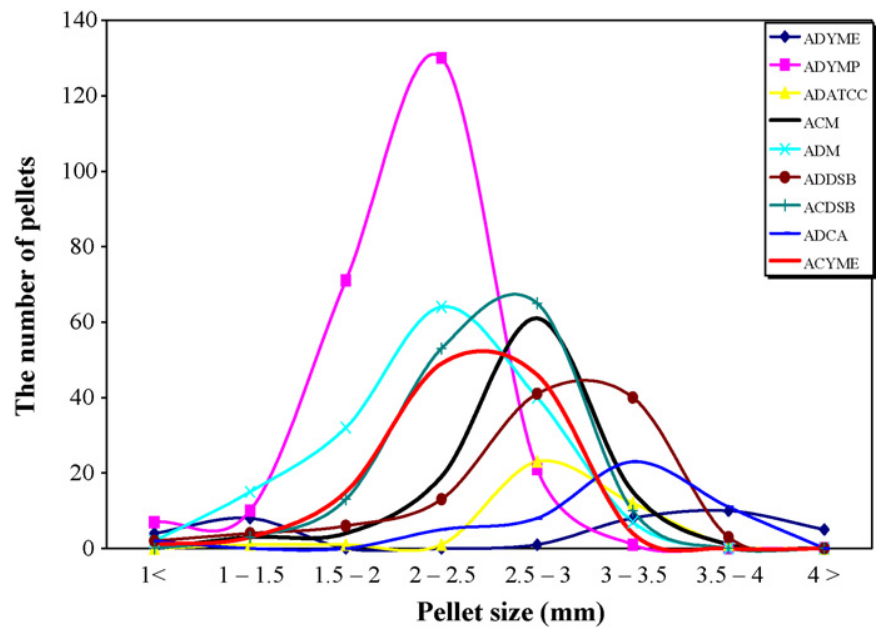

Fig. 6. Pellet size distribution of A. sojae in shake flask experiments resulted in pellet type of morphology. Explanation of the abbreviations are given in Table 4. 
media, would result in maximum polygalacturonase activity and desired pellet morphology under the conditions studied.

\section{Conclusions}

A full factorial statistical analysis revealed that factors of strain types and fermentation media and the interaction between them was significant on the polygalacturonase enzyme activity, whereas, the seed culture compositions were found insignificant at the significance level of $1 \%$. However, the seed culture composition had profound influence on the spore production and morphology of the final fermentation broth affecting the rheology and consequently the downstream processing. It was determined, that $A$. sojae grown on molasses and inoculated into complex media resulted in pellet morphology, and Newtonian type of broth rheology, in the production of polygalacturonase enzyme. This study demonstrated also that, A. sojae, known mainly in solid state fermentation of the popular koji food, could be a good candidate in submerged fermentations, with the desired morphological characteristics, in the production of the polygalacturonase as well as other potential enzymes.

\section{Acknowledgement}

Financial support of Izmir Institute of Technology through the projects IYTE 2004, 04 and IYTE 2004, 08 is gratefully acknowledged.

\section{References}

[1] A. Mitard, J.P. Riba, Morphology and growth of Aspergillus niger ATCC 26036 cultivated at several shear rates, Biotechnol. Bioeng. 32 (1987) 835-840.

[2] Z.J. Li, V. Shukla, A.P. Fordyce, A.G. Pedersen, K.S. Wenger, M.R. Marten, Fungal morphology and fragmentation behavior in a fed-batch Aspergillus oryzae fermentation at the production scale, Biotechnol. Bioeng. 70 (2000) 300-312.

[3] M. Pazauki, T. Panda, Understanding the morphology of fungi, Bioprocess Eng. 22 (2000) 127-143.

[4] U. Reichl, R. King, E.D. Gilles, Characterization of pellet morphology during submerged growth of Streptomyces tendae by image analysis, Biotechnol. Bioeng. 39 (1991) 164-170.

[5] Y. Zhaou, J. Du, G.T. Tsao, Mycelial pellet formation by Rhizopus oryzae ATCC 20344, Appl. Biochem. Biotechnol. 84-86 (2000) 779-789.

[6] B. Atkingson, L.S. Daoud, Microbial flogs and flocculation in fermentation process engineering, Adv. Biochem. Eng. 4 (1976) 41-124.

[7] B. Metz, N.W.F. Kossen, The growth of molds in the form of pellets. A literature review, Biotechnol. Bioeng. 19 (1977) 781-799.

[8] M. Papagianni, Fungal morphology and metabolite production in submerged mycelial processes, Biotechnol. Adv. 22 (2004) 189-259.
[9] D. Ryoo, Fungal fractal morphology of pellet formation in Aspergillus niger, Biotechol. Tech. 13 (1999) 33-36.

[10] G. Kaur, S. Kumar, T. Satyanaraya, Production and characterization and application of a thermostable polygalacturonase of a thermophilic mould Sporotrichum thermophile Apinis, Bioresour. Technol. 94 (2004) 239243.

[11] M. Puri, A. Banerjee, U.C. Banerjee, Optimization of process parameters for the production of naringinase by Aspergillus niger MTCC 1344, Process Biochem. 40 (2005) 195-201.

[12] M. Elibol, Optimization of medium composition for actinorhodin production by Streptomyces coelicolor A3 (2) with response surface methodology, Process Biochem. 39 (2004) 1057-1062.

[13] S.J. Tellez, A.B. Moldes, J.L. Alonso, M. Vazquez, Optimization of lactic acid production by Lactobacillus delbuecki through response surface methodology, J. Food Sci. 68 (2003) 1454-1458.

[14] K. Saito, N. Takakuwa, Y. Oda, Purification of the extracellular pectinolytic enzyme from the fungus Rhizopus oryzae NBRC 4707, Microbiol. Res. 159 (2004) 83-86.

[15] K. Saito, Y. Kawamura, Y. Oda, Role of the pectoloytic enzyme in the lactic acid fermentation of potato pulp by Rhizopus oryzae, J. Ind. Microbiol. Biotechnol. 30 (2003) 440-444.

[16] J.W. Bennett, Aspergillus and Koji: history, practice and molecular biology, Soc. Ind. Enzymes News 51 (2001) 65-71.

[17] T. Panda, G.S.N. Naidu, J. Sinha, Multiresponse analysis of microbiological parameters affecting the production of pectolytic enzymes by Aspergillus niger: a statistical view, Process Biochem. 35 (1999) 187195.

[18] A.B. Metzner, Rheology of suspensions in polymeric liquid, Soc. Rheol. 29 (6) (1985) 739-775.

[19] P.W. Cox, C.R. Thomas, Classification and measurement of fungal pellets by automated image analysis, Biotechnol. Bioeng. 39 (1992) 945952.

[20] J.L.C. Lopez, J.A.S. Perez, J.M.F. Sevilla, E.M.J. Porcel, Y. Chisti, Pellet morphology, culture rheology and lovastatin production in cultures of Aspergillus terreus, J. Biotechnol. 116 (2005) 61-77.

[21] C.R.D. Pamboukian, M.C.R. Facciotti, W. Schmidell, Relationship between morphology, rheology and glucoamylase production by Aspergillus awamori in submerged cultures, Braz. J. Chem. 15 (1998) 1-8.

[22] G.S. Bryne, O.P. Ward, Effect of nutrition on pellet formation by Rhizopus arrhizus, Biotechnol. Bioeng. 33 (1989) 912-914.

[23] C.T. Calam, Starting investigational and production cultures, Process. Biochem. 11 (1976) 1-7.

[24] G.S. Naidu, T. Panda, Application of response surface methodology to evaluate some aspects on stability of pectolytic enzymes from Aspergillus niger, Biochem. Eng. J. (1998) 71-77.

[25] A. Blandino, K. Dravillas, D. Cantero, S.S. Pandiella, C. Webb, Utilisation of whole flour for the production of extracellular polygalacturonases by some fungal strains, Process Biochem. 37 (2001) 497-503.

[26] P. Znidarsic, R. Komel, A. Pavko, Influence of some environmental factors on Rhizopus nigricans submerged growth in the form of pellets, World J. Microbiol. Biotechnol. 16 (2000) 589-593.

[27] L.X. Du, S.J. Jia, F.P. Lu, Morphological changes of Rhizopus chinesis 12 in submerged culture and its relationship with antibiotic production, Process Biochem. 38 (2003) 1643-1646.

[28] Difco and BBL Manual, Manual of Microbiological Culture Media, Becton Dickonson and Company, Sparks, Maryland, 2003. 